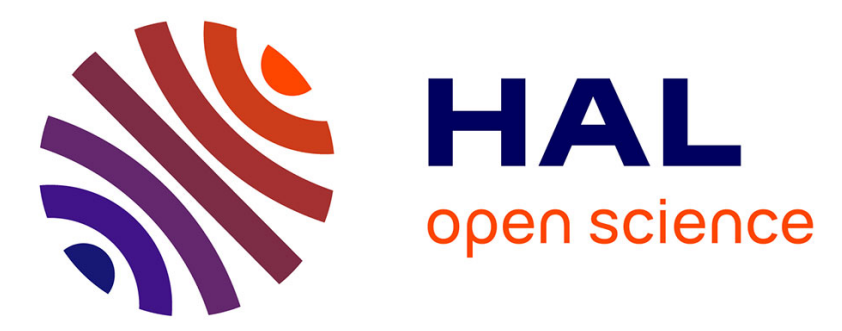

\title{
Characterization of Chemical Bonding in Ion-Implanted Polymers by Means of Mid-Infrared Reflectivity
}

\author{
V.G. Ivanov, G. Hadjichristov, Eric Faulques
}

\section{To cite this version:}

V.G. Ivanov, G. Hadjichristov, Eric Faulques. Characterization of Chemical Bonding in Ion-Implanted Polymers by Means of Mid-Infrared Reflectivity. Applied Spectroscopy, 2009, 63 (9), pp.1022. 10.1366/000370209789379286 . hal-00432062

\section{HAL Id: hal-00432062 \\ https://hal.science/hal-00432062}

Submitted on 30 Apr 2020

HAL is a multi-disciplinary open access archive for the deposit and dissemination of scientific research documents, whether they are published or not. The documents may come from teaching and research institutions in France or abroad, or from public or private research centers.
L'archive ouverte pluridisciplinaire HAL, est destinée au dépôt et à la diffusion de documents scientifiques de niveau recherche, publiés ou non, émanant des établissements d'enseignement et de recherche français ou étrangers, des laboratoires publics ou privés. 


\title{
Characterization of Chemical Bonding in Ion-Implanted Polymers by Means of Mid-Infrared Reflectivity
}

\author{
VICTOR G. IVANOV,* GEORGI HADJICHRISTOV, and ERIC FAULQUES \\ Sofia University, Faculty of Physics, 5 James Bourchier Blvd., 1164 Sofia, Bulgaria (V.G.I.); Georgi Nadjakov Institute of Solid State Physics, \\ Bulgarian Academy of Sciences, 72 Tzarigradsko Chaussee Blvd., 1784 Sofia, Bulgaria (G.H.); and Institut des Matériaux Jean Rouxel, \\ UMR6502 CNRS, Nantes Atlantic Universities, 2 rue de la Houssiniere - BP 32229, 44322 Nantes, France (E.F.)
}

\begin{abstract}
An optical approach for structural characterization of the modified surface layer in ion-implanted polymers is proposed. The mid-infrared reflectivity from the implanted surface is analyzed in terms of an oscillator dispersion model combined with the theory of differential reflection spectroscopy. The degree of destruction of a specific chemical bond is determined by the relative drop of the oscillator strengths associated with the corresponding vibrational modes. As an example, this methodology is applied to poly(methylmethacrylate) (PMMA) implanted with $50 \mathrm{keV}$ silicon ions at fluences in the range $3 \times 10^{14}$ to $1 \times 10^{17}$ ions/ $\mathrm{cm}^{2}$. The scission rates for the $\mathrm{C}=\mathrm{O}, \mathrm{C}-\mathrm{O}-\mathrm{C}$, and $\mathrm{C}-\mathrm{H}$ bonds, as well as the static dielectric constant of the ion-modified material, are calculated as a function of the ion fluence. Further, a lower-limit estimate of $120 \mathrm{~nm}$ for the thickness of the ion-modified layer is obtained.
\end{abstract}

Index Headings: Ion-implanted polymers; Fourier transform infrared spectroscopy; FT-IR; Differential reflectance spectroscopy; DRS; Poly(methylmethacrylate); PMMA.

\section{INTRODUCTION}

Ion implantation strongly modifies the mechanical, electrical, and optical properties of polymer surfaces. ${ }^{1,2}$ A common structural modification of the ion-implanted hydrocarbon polymers is the formation of a hydrogenated amorphous carbon (HAC) layer with a predominant graphite-like $\mathrm{sp}^{2}$ bonding. This carbonaceous layer is responsible for the strong enhancement of the surface conductivity of the ion-implanted polymeric materials. The possibility to control the type, the energy, and the fluence of the implantation ions allows for controllable fabrication of polymer-based electronic devices. ${ }^{3-6}$

The average size of the $\mathrm{sp}^{2}$ HAC domains and their hydrogen content can be efficiently assessed by means of Raman spectroscopy in the visible range. ${ }^{7-9}$ The excitation in this spectral range, e.g., at $\lambda=514 \mathrm{~nm}$, is resonant with the electronic transitions around the $M$-point of the Brilloin zone of graphite, leading to a significant enhancement of the characteristic $G$ - and $D$-bands of HAC ${ }^{10,11}$ over the polymer's intrinsic Raman-active lines. Thus, the Raman signal from the polymer is strongly masked by the intense HAC bands as well as by a broad photoluminescence background and may not provide adequate information for the changes of chemical bonding in the polymer provoked by the implantation ions.

The infrared spectroscopic techniques are more sensitive to the variations of the chemical bonding in the ion-implanted and ion-irradiated polymers, since the $D$ - and $G$-bands of graphite phase correspond to centrosymmetric modes and do not posses infrared activity. We will mention only two instructive examples. The attenuated total reflection (ATR) from 1350 $\mathrm{keV}$ proton-irradiated poly(methylmethacrylate) (PMMA) ${ }^{12}$

Received 22 December 2008; accepted 22 June 2009.

* Author to whom correspondence should be sent. E-mail: vgi@phys. uni-sofia.bg. reveals a significant decrease of the infrared absorption bands of the polymer, which is interpreted as a result of decomposition of the pendant ester and methyl groups. Also, the infrared absorption spectroscopy has been efficiently used to characterize the decomposition of chemical bonds in latent tracks produced by $\mathrm{MeV}$ light ions in plastic track detectors. ${ }^{13}$

$\mathrm{X}$-ray photoelectron spectroscopy (XPS) is another sensitive probe for chemical modifications of the surface. However, being sensitive to the top surface layer of a few nanometers, the XPS technique is not very informative for the buried (to a depth more than $50 \mathrm{~nm}$ ) ion implanted layer. Generally, more suitable are the depth-profiling measurements when XPS is accompanied by ion sputtering of the sample using low-energy ions (e.g., several keV noble gas ions) for sample erosion. In the polymers sensitive to ion impacts, however, the information about chemical bonding obtainable from XPS is perturbed by the chemical and structural changes during the sputtering itself.

The aim of this work is to extend the capabilities of midinfrared (MIR) spectroscopy for nondestructive quantitative characterization of the chemical bonding in ion-implanted polymers. We propose an approach based on differential reflection spectroscopy (DRS) ${ }^{14-16}$ in the MIR region combined with the Lorentz dispersion model. To our best knowledge, such a spectroscopic characterization has not been used for ion-implanted polymers. In contrast to ATR, which is sensitive to a surface region of a thickness $d$ comparable to the radiation wavelength $\lambda$, DRS provides information for very thin layers of thickness $d \ll \lambda$, which is the case for the ionmodified layer of the ion-implanted polymers. The theoretical background of the method is described in the next section. In the third section we report and analyze the results obtained by this methodology for PMMA implanted with $50 \mathrm{keV} \mathrm{Si}{ }^{+}$ions at fluences in the range of $3 \times 10^{14}$ to $1 \times 10^{17}$ ions $/ \mathrm{cm}^{2}$. The main conclusions of the study are summarized in the final section.

\section{THEORETICAL BACKGROUND}

The MIR dielectric function in dielectrics includes two contributions: a monotonous non-resonant part $\varepsilon_{\mathrm{nr}}(\omega)$ originating from high-energy (optical) electronic transitions across the gap and a resonant part due to the polarization response of the interatomic vibrations. The non-resonant part is usually approximated by a constant: $\varepsilon_{\mathrm{nr}}(\omega)=\varepsilon_{\infty}$, the high-frequency (optical) dielectric constant. The resonant contribution from the vibrational modes can be approximated by a sum of Lorentz oscillator (LO) response functions. Thus, the dielectric function takes the form

$$
\varepsilon(\omega)=\varepsilon_{\infty}+\sum_{i=1}^{n} \frac{f_{i} \omega_{i}^{2}}{\omega_{i}^{2}-\omega^{2}-i \gamma_{i} \omega}
$$


where $\omega_{i}$ is the resonant frequency of the $i$ th vibration, and $\gamma_{i}$ is its damping constant, related to the full width at half-maximum (FWHM) of the corresponding band in the infrared absorption spectrum. The dimensionless oscillator strength $f_{i}$ is the contribution of the $i$ th oscillator to the static dielectric constant of the material, i.e.:

$$
\varepsilon_{0}=\varepsilon_{\infty}+\sum_{i=1}^{n} f_{i}
$$

The reflectivity at normal incidence is linked to the dielectric function through the Fresnel's formula:

$$
R(\omega)=\left|\frac{\sqrt{\varepsilon(\omega)}-1}{\sqrt{\varepsilon(\omega)}+1}\right|^{2}
$$

Thus, oscillator parameters can be determined by fitting Eq. 3 to the experimental reflectivity spectrum. This procedure will be referred to hereafter as the LO fit.

When a substrate with a dielectric function $\varepsilon_{S}(\omega)$ is coated by a thin layer of material, whose dielectric function $\varepsilon_{\mathrm{L}}(\omega)$ differs from $\varepsilon_{S}(\omega)$, the reflectivity spectrum (Eq. 3) modifies to $R_{\text {mod }}(\omega)$. The differential reflectance (DR) is defined as $\left(R_{\text {mod }}-\right.$ $R) / R \equiv \Delta R / R$. The exploration of DR allows a wealth of qualitative and quantitative information to be obtained for the surface layer and has been widely used for characterization of the surface modifications of semiconductors ${ }^{17}$ or for thin polymer layers on different substrates. ${ }^{18-20}$ In the approximation of a semi-infinite substrate and a layer thickness $d$ much smaller than the wavelength $\lambda$ of the incident radiation, the linearization of Fresnel equations ${ }^{14}$ gives a simple formula for DR:

$$
\frac{\Delta R}{R}=8 \pi \bar{v} d \operatorname{Im}\left(\frac{\varepsilon_{\mathrm{L}}-1}{\varepsilon_{\mathrm{S}}-1}\right)
$$

In this equation $\bar{v}=1 / \lambda=\omega /(2 \pi c)$ is the wavenumber of the incident radiation. The prefactor $8 \pi \bar{v} d$ accounts for the phase shift between waves reflected directly from the layer and from the layer-substrate interface. It is assumed in Eq. 4 that the complex dielectric function is represented in the form: $\varepsilon=\varepsilon^{\prime}+$ $i \varepsilon^{\prime \prime}$. Combining Eqs. 3 and 4, one obtains an expression for the normal-incidence reflectance of the layer-substrate structure:

$$
R_{\text {mod }}(\omega)=\left|\frac{\sqrt{\varepsilon_{\mathrm{S}}(\omega)}-1}{\sqrt{\varepsilon_{\mathrm{S}}(\omega)}+1}\right|^{2}\left[1+8 \pi \bar{v} d \operatorname{Im} \frac{\varepsilon_{\mathrm{L}}(\omega)-1}{\varepsilon_{\mathrm{S}}(\omega)-1}\right]
$$

The condition $d \ll \lambda$ is satisfied in the case of MIR reflectivity from ion-implanted polymers since the thickness of the ion-modified layer reaches typically several hundreds of nanometers, while the wavelengths of the MIR radiation are at least one order of magnitude greater. In this case $\varepsilon_{S}$ refers to the dielectric function of the bare polymer material, while $\varepsilon_{\mathrm{L}}=(1 /$ d) $\int_{0}^{d} \varepsilon(z) \mathrm{d} z$ expresses the average dielectric function in the ion-modified layer.

Provided that the dielectric function $\varepsilon_{S}(\omega)$ is extracted from a LO fit to the reflectivity spectrum of the non-implanted material, Eq. 5 couples both the real and imaginary parts of the dielectric function $\varepsilon_{\mathrm{L}}(\omega)$ of the modified layer. In order to determine the two parts separately, numerical approaches based on the Kramers-Kronig relations in the visible range have been proposed. ${ }^{18-20}$ However, these methods require that the nonresonant part of $\varepsilon_{\mathrm{L}}(\omega)$ is practically constant in the spectral range of interest. As will be shown in the next section, this is not the case for the ion-implanted polymers due to the strong absorption of MIR radiation in the ion-modified layer. Here we model the dielectric function $\varepsilon_{\mathrm{L}}(\omega)$ by a sum of Lorentz oscillators in order to fit Eq. 5 to the reflectivity spectrum of the implanted material. In this way, one can extract both the average oscillator strengths of the corresponding vibrational modes and the average high-frequency (or static) dielectric constant of the ion-modified layer. Hereafter we will refer to this procedure as the $\mathrm{LO}+\mathrm{DR}$ fit. It has to be emphasized that the layer thickness $d$ can not be extracted as an independent variable from the $\mathrm{LO}+\mathrm{DR}$ fit. In order to determine the oscillator parameters one must impose a priori a definite value of $d$ based either on independent experiments or on theoretical estimates. In our work we use values for $d$ obtained from SRIM simulations ${ }^{21}$ of the $\mathrm{Si}^{+}$stopping range in PMMA. Alternatively, as will be explained in the next section, the LO+DR fit itself can provide a lower-limit estimate for $d$.

The most important parameters in our methodology are the oscillator strengths. In a first approximation they are proportional to the volume concentration of the corresponding oscillators. Thus, the change of the oscillator strength of a given MIR band can be attributed to the change of the concentration of the chemical bonds (or functional groups) involved in that vibration. Accordingly, we introduce an average "scission rate" $S$ for a given bond (group) as:

$$
S(\Phi)=\frac{\sum_{i}\left[f_{i}(0)-f_{i}(\Phi)\right]}{\sum_{i} f_{i}(0)}
$$

Here the sum runs over the characteristic vibrations of the chemical bond or functional group considered. The quantities $f_{i}(0)$ are the oscillator strengths of the corresponding vibrations in the non-implanted material as obtained by the LO fit. The quantities $f_{i}(\Phi)$ are the average oscillator strengths of the same vibrations in the ion-modified material at an ion fluence $\Phi$, as extracted by the LO+DR fit. The scission rate is a metric for the extent of bond breaking in a surface layer of a thickness $d$.

\section{EXPERIMENTAL RESULTS AND DISCUSSION}

We applied the above described methodology to probe the structural decomposition of PMMA, implanted with $\mathrm{Si}^{+}$ions of energy $50 \mathrm{keV}$ and ion fluences in the range of $3 \times 10^{14}$ to $1 \times$ $10^{17}$ ions $/ \mathrm{cm}^{2}$. The details of sample preparation are described elsewhere. ${ }^{9}$ UV-visible-NIR specular reflection measurements ${ }^{9}$ from the implanted surface reveal well-pronounced interference oscillations as a function of $\lambda$, which are signatures of a good optical quality of the surface. In addition, measurements in an integrating sphere show that the diffuse reflectance decreases steadily from $5 \%$ to less then $1 \%$ when the wavelength increases from 800 to $2500 \mathrm{~nm}$. It suggests that in the MIR range $\left(\lambda \sim 10^{4} \mathrm{~nm}\right)$, the diffuse reflection is much less significant then the specular reflection. Reference Raman spectra were taken at an excitation wavelength of $633 \mathrm{~nm}$ (HeNe laser) on a LabRAM (Horiba Jobin-Yvon) spectrophotometer. The MIR specular reflectance spectra were obtained on a Bruker Vertex 70 Fourier transform infrared (FT-IR) spectrometer at an angle of incidence of $10^{\circ}$, which allows a 


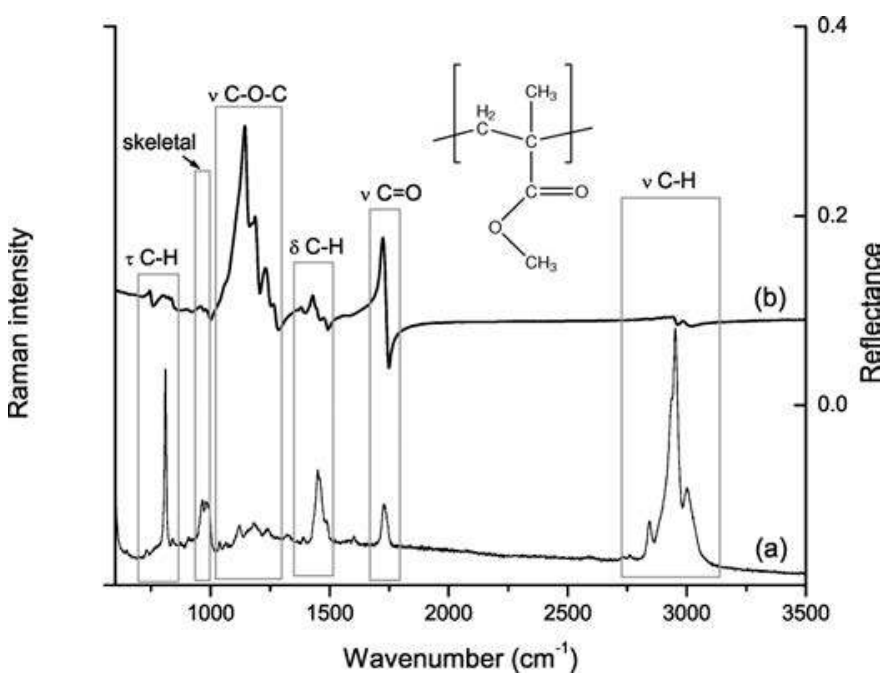

FIG. 1. Comparison between (a) the Raman spectrum and (b) the MIR reflectivity spectrum of pure PMMA. The chemical structure of the PMMA repeating unit is shown in the inset.

nearly normal-incidence mode of reflection to be considered. The sample thickness of $5 \mathrm{~mm}$ was sufficient to eliminate the reflection from the rear surface due to the strong absorption of the MIR radiation into the material.

Shown in Figs. 1a and $1 \mathrm{~b}$ are a Raman spectrum and a reflectivity spectrum, respectively, of the non-implanted PMMA material. The PMMA vibrational modes observed in the Raman scattering appear as well-resolved dispersion features in the reflectivity spectrum. The high-frequency modes in the range 2800 to $3000 \mathrm{~cm}^{-1}$ stem from $\mathrm{C}-\mathrm{H}$ stretching vibrations. They are very intense in the Raman spectrum but weakly pronounced in MIR reflectivity. The double-bond $\mathrm{C}=\mathrm{O}$ stretching vibration at $1730 \mathrm{~cm}^{-1}$ is very strong in the MIR spectrum due to the high polarity of that bond. The lines in the 1370 to $1450 \mathrm{~cm}^{-1}$ range are characteristic for the $\mathrm{C}-\mathrm{H}$ bending vibrations of the methyl $\mathrm{CH}_{3}$ and methylene $\mathrm{CH}_{2}$ groups. The modes in the 1000 to $1240 \mathrm{~cm}^{-1}$ range correspond to single-bond $\mathrm{C}-\mathrm{O}-\mathrm{C}$ stretching vibrations of the ester groups. Again, due to highly polar character of the $\mathrm{C}-\mathrm{O}$ bonds, these modes possess very strong IR activity. The low frequency part of the vibrational spectra is dominated by the $\mathrm{CH}_{2}$ and $\mathrm{CH}_{3}$ rocking modes $\left(\sim 750 \mathrm{~cm}^{-1}, \sim 810 \mathrm{~cm}^{-1}\right)$ and the carbon vibrations of the polymer skeleton $\left(\sim 960-990 \mathrm{~cm}^{-1}\right)$.

Figure 2 compares the reflectivity spectra obtained from PMMA samples implanted at different fluences with the reflectivity spectrum of the non-implanted PMMA. The most evident spectral modifications due to implantation are (1) a significant reduction of the vibrational dispersion features, which is most pronounced for the $\mathrm{C}=\mathrm{O}$ stretching mode at $1730 \mathrm{~cm}^{-1}$; and (2) appearance of a featureless background, which increases monotonically with frequency.

The diminution of the vibrational dispersion features in the implanted samples is attributed to the decrease of the oscillator strengths of the corresponding vibrations due to the bond scission in the polymer. In order to understand the origin of the reflectivity background, we will analyze Eq. 4 for DR. The background emerges in a frequency range where the pure polymer exhibits no sharp resonant features, i.e., the dielectric function $\varepsilon_{S}(\omega)$ is nearly constant. In that case, the differential reflectance is proportional to the imaginary part of the dielectric

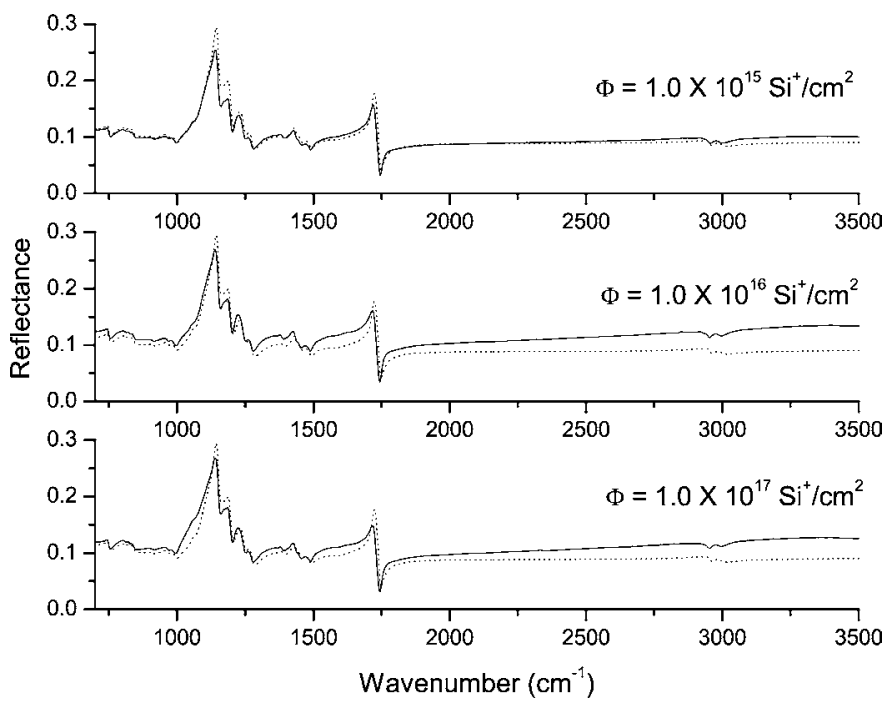

FIG. 2. Normal incidence MIR reflectivity of PMMA implanted at different $\mathrm{Si}^{+}$fluences (the solid lines). The reflectivity spectrum of non-implanted PMMA is given in all panels for comparison (the dotted lines).

function of the surface layer:

$$
\frac{\Delta R}{R}=\frac{8 \pi d}{\varepsilon_{\mathrm{S}}-1} \bar{v} \varepsilon_{\mathrm{L}}^{\prime \prime}
$$

Therefore, the reflectivity background of the implanted samples can be considered as a result of an absorption in the ion-modified layer in a wide frequency range. The absorption takes place most probably in the carbon nanoclusters where low-energy direct optical transitions are allowed around the $M$ point of the Brilloin zone of graphite. For that reason, in the further analysis the non-resonant part of the dielectric function $\varepsilon_{L}(\omega)$ is taken up to the linear (imaginary) term of the frequency series expansion:

$$
\varepsilon_{\mathrm{nr}}(\omega)=\varepsilon_{\infty}+i A \omega
$$

where the coefficient $A$ is an adjustable parameter.

Figure 3 illustrates the distribution functions for carbon, oxygen, and hydrogen recoil atoms as well as that of $\mathrm{Si}$ ions, as obtained from SRIM calculations for $\mathrm{Si}^{+}$energy of $50 \mathrm{keV}$. The distribution functions were collected on the basis of individual atomic tracks produced by $10^{5} \mathrm{Si}^{+}$ions. It is seen that the recoil-atom and ion distributions extend up to approximately $1700 \AA$. Thus, a value of $d=170 \mathrm{~nm}$ will be adopted in our analysis.

The LO fit to the reflectivity spectrum of the non-implanted material is shown in Fig. 4a. The extracted values of the resonant frequencies, their FWHM, and the oscillator strengths were used to calculate the dielectric function $\varepsilon_{S}(\omega)$ of the nonimplanted polymer. Then LO+DR fit to the reflectivity spectra of the implanted samples was applied by fixing the oscillator frequencies and FWHM and varying the oscillator strengths and the real and imaginary parts of the non-resonant part (see Eq. 8) of the dielectric function $\varepsilon_{\mathrm{L}}(\omega)$. The fitted reflectivity spectrum corresponding to a fluence of $1 \times 10^{17}$ ions $/ \mathrm{cm}^{2}$ is presented in Fig. 4b.

Shown in Fig. 5a are the variations of the scission rate, as defined by Eq. 6 , as a function of the ion fluence. The $\mathrm{C}-\mathrm{H}$ scission rate was calculated on the basis of a sum of oscillator 


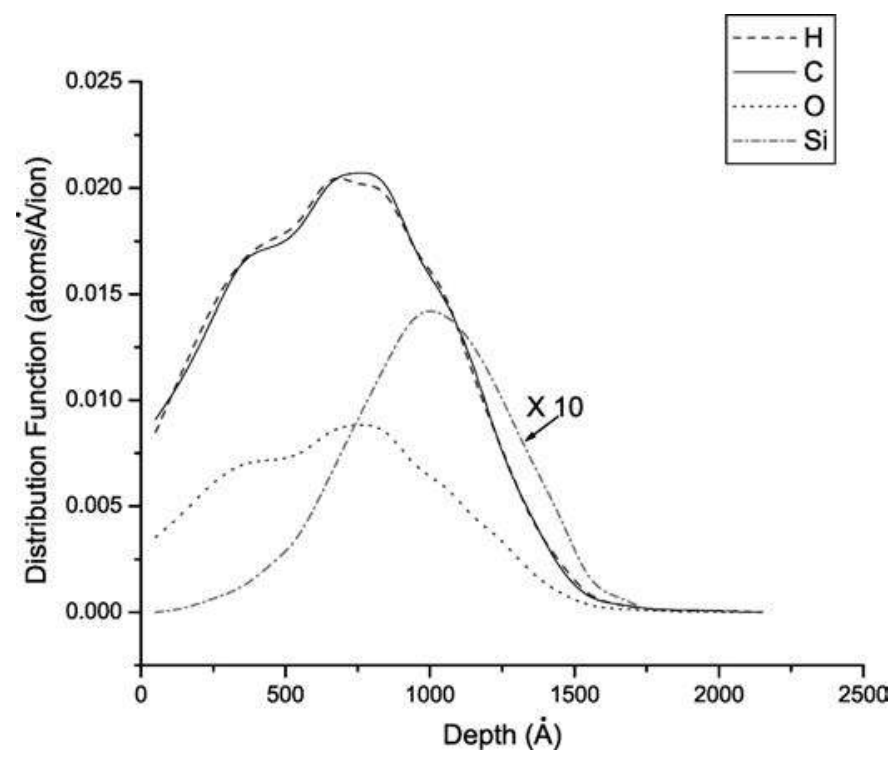

FIG. 3. In-depth atomic distribution functions for hydrogen, carbon, oxygen, and silicon (the latter multiplied by a factor of 10 ).

strengths of the $\mathrm{C}-\mathrm{H}$ stretching vibrations in the 2800-3000 $\mathrm{cm}^{-1}$ interval and the $\mathrm{C}-\mathrm{H}$ bending vibrations in the range $1370-1450 \mathrm{~cm}^{-1}$. For the $\mathrm{C}-\mathrm{O}-\mathrm{C}$ groups the vibrational modes in the range $1000-1240 \mathrm{~cm}^{-1}$ were taken into account, while for the double $\mathrm{C}=\mathrm{O}$ bond only the $1730 \mathrm{~cm}^{-1}$ mode was considered.

The results indicate that the bond destruction of the main functional groups varies non-monotonically with the ion fluence. At fluences up to $1 \times 10^{16} \mathrm{Si}^{+} / \mathrm{cm}^{2}$, the effect of the ion implantation for the bond scission is evident and most pronounced for the double $\mathrm{C}=\mathrm{O}$ bond. At higher fluences, however, various competitive processes take place, e.g., thermal annealing, which result in a decrease of the scission rate for $\mathrm{C}=\mathrm{O}$ and $\mathrm{C}-\mathrm{H}$ bonds in the methyl groups. The relatively low value of the scission rate for the $\mathrm{C}-\mathrm{O}-\mathrm{C}$ groups around $1 \times 10^{15}$ ions $/ \mathrm{cm}^{2}$ can be explained by recovery processes provoked by carbonyl group destruction and a subsequent bridging of the oxygen atom to another carbon atom: $\mathrm{C}=\mathrm{O}+\mathrm{C} \rightarrow \mathrm{C}-\mathrm{O}-\mathrm{C}$. It is instructive to note that our estimates of the scission rates agree well with XPS studies of $\mathrm{Si}^{*}$ implanted PMMA at comparable ion energies. ${ }^{22,23}$ Particularly, these works support the conjecture that the implanted ions interact more strongly and break more efficiently the double $\mathrm{C}=\mathrm{O}$ bonds than the $\mathrm{C}-\mathrm{O}-\mathrm{C}$ groups.

The static dielectric constant of the non-implanted PMMA was estimated to be $\varepsilon_{0}=3.62 \pm 0.04$ from the LO fit. Figure $5 \mathrm{~b}$ represents the dependence of the fitted static dielectric constant of the ion-modified layer on the ion fluence. Upon increasing the ion fluence, $\varepsilon_{0}$ follows a trend reciprocal to that of the scission rate for the double $\mathrm{C}=\mathrm{O}$ bond. This behavior can be explained by the fact that the most significant contribution to the dielectric constant comes from the highly polar $\mathrm{C}=\mathrm{O}$ bonds and, therefore, their diminution leads to a decrease of $\varepsilon_{0}$.

As mentioned above, our calculations of the scission rates are based on an independent estimate $d=170 \mathrm{~nm}$ for the thickness of the ion-modified layer. If the real thickness differs from that estimate, then all the values of the scission rate should be recalculated in an inverse proportion to the real value of $d$. Since the scission rate for a specific bond can not exceed 1

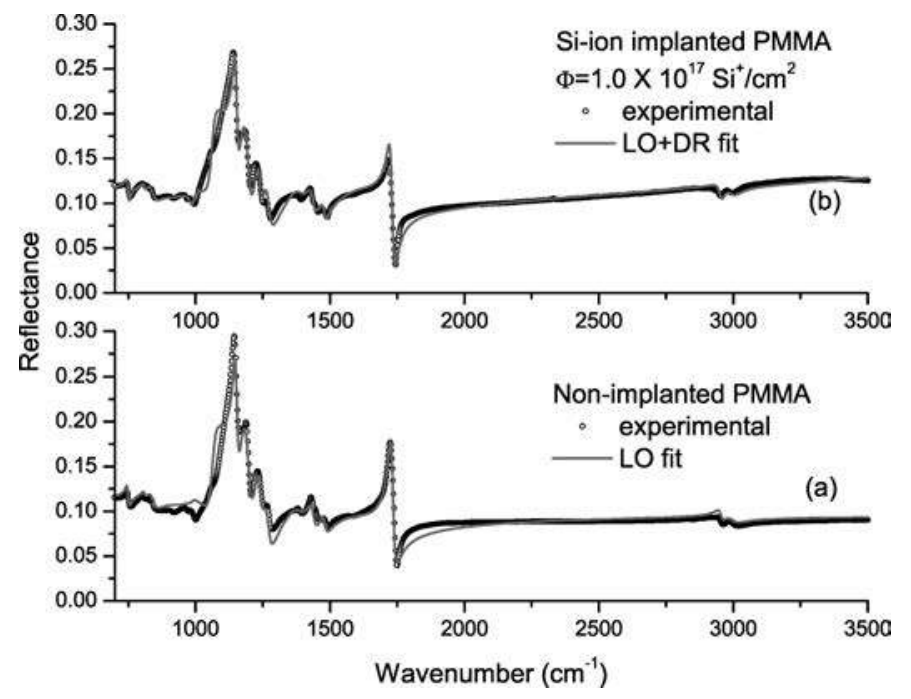

FIG. 4. (a) LO fit to the MIR reflectivity spectrum of the pure PMMA plastic; (b) LO+DR fit to the reflectivity spectrum of the material implanted at a fluence of $1 \times 10^{17} \mathrm{Si}^{+} / \mathrm{cm}^{2}$.

(this case corresponds to a complete lack of infrared activity of the associated vibrational modes, i.e., zero oscillator strength) a lower limit for the thickness $d$ can be estimated. From Fig. 5a we see that for $d=170 \mathrm{~nm}$ a maximum scission rate of 0.68 is calculated for the double $\mathrm{C}=\mathrm{O}$ bond at a fluence of $1 \times 10^{16}$ ions $/ \mathrm{cm}^{2}$. Thus, we conclude that the lower limit for the thickness of the implanted layer is $d_{\min }=120 \mathrm{~nm}$ since at this value the scission rate for that bond would reach a value of 1.0. It is clear, however, that the relative sizes of scission rates for different bonds are invariant with respect to the adopted value of $d$.

\section{CONCLUSION}

Ion implantation has a sizable effect on the vibrational features in the MIR reflectivity spectra of organic polymers.

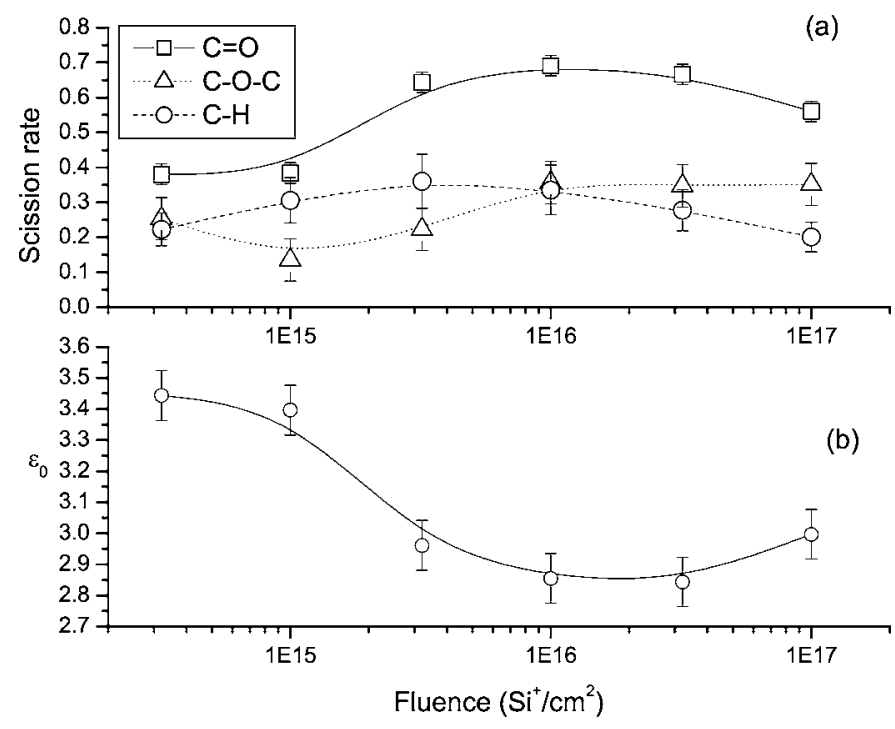

FIG. 5. (a) Scission rates for the $\mathrm{C}=\mathrm{O}, \mathrm{C}-\mathrm{H}$, and $\mathrm{C}-\mathrm{O}-\mathrm{C}$ groups; (b) the static dielectric constant $\varepsilon_{0}$ as a function of the ion fluence. The solid curves are guides to the eye. 
The modifications of the reflectivity spectra upon ion implantation can be analyzed in terms of differential reflectance theory combined with the Lorenz oscillator model. We demonstrate that at a variety of implantation fluences, the degree of bond scission of distinct functional groups of the polymer can be deduced in terms of the relative reduction of the oscillator strengths relevant to the corresponding vibrational modes. Besides monitoring of the chemical bonding, the proposed approach is applicable to optically characterize the electronic properties of the ion-modified layer, particularly by estimation of the static dielectric constant.

In our research, this technique is tested on a specific polymer, PMMA, but the methodology is quite general and can be applied to a wide range of materials and a wide range of accelerated ions. The proposed technique is sensitive to both components (real and imaginary) of the dielectric response of the ion-modified material. Similarly to other spectroscopy techniques, e.g., diffuse reflection spectroscopy, the subsurface absorption (the imaginary part of the complex dielectric constant) within samples is efficiently probed. The sensitivity and chemical selectivity of the approach proposed here make it well suited to nondestructive inspection of ion-modified polymeric materials.

\section{ACKNOWLEDGMENTS}

This work was supported by the contracts FNI 092/2009 of the Sofia University and D01-835/2007 of the Ministry of Education of Bulgaria.

1. D. V. Sviridov, V. B. Odzhaev, and I. P. Kozlov, Electrical and Optical Polymer Systems - Fundamentals, Methods and Applications, D. L. Wise, G. E. Wnek, D. J. Trantolo, T. M. Cooper, and J. D. Gresser, Eds. (Marcel Dekker, New York, 1998), p. 387.
2. D. Fink, Fundamentals of Ion Irradiated Polymers (Springer-Verlag, Berlin, 2004).

3. P. Mazzoldi and G. W. Arnold, Ion Beam Modification of Insulator (Elsevier, Amsterdam, 1987).

4. D. Fink, Transport Processes in Ion-Irradiated Polymers (Springer-Verlag, Berlin, 2004).

5. R. E. Giedd, M. G. Moss, J. Kaufmann, and Y. Q. Wang, Electrical and Optical Polymer Systems - Fundamentals, Methods and Applications, D. L. Wise, G. E. Wnek, D. J. Trantolo, T. M. Cooper, and J. D. Gresser, Eds. (Marcel Dekker, New York, 1998), p. 1011.

6. V. N. Popok, I. A. Karpovich, V. B. Odzhaev, and D. V. Sviridov, Nucl. Instrum. Meth. B 148, 1106 (1999).

7. E. H. Lee, D. M. Hembree, G. R. Rao, and L. K. Mansur, Phys. Rev. B 48 15540 (1993).

8. A. Das, S. Dhara, and A. Patnaik, Phys. Rev. B 59, 11069 (1999).

9. G. B. Hadjichristov, V. G. Ivanov, and E. Faulques, Appl. Surf. Sci. 254, 4820 (2008).

10. A. Ferrari and J. Robertson, Phys. Rev. B 61, 14095 (1999).

11. C. Casiraghi, A. Ferrari, and J. Robertson, Phys. Rev. B 72, 085401 (2005).

12. H. W. Choi, H. J. Woo, W. Hong, J. K. Kim, S. K. Lee, and C. H. Eum, Appl. Surf. Sci. 169-170, 433 (2001).

13. R. Barillon, M. From, R. Katz, and A. Chambaudet, Rad. Prot. Dosim. 99 359 (2002).

14. J. D. E. McIntyre and D. E. Aspnes, Surf. Sci. 24, 417 (1971).

15. P. Chiarada and R. Del Sole, Surf. Rev. Lett. 6, 517 (1999).

16. Y. Borensztein, Surf. Rev. Lett. 7, 399 (2000).

17. Y. Borensztein, Phys. A 207, 293 (1994).

18. R. Nitsche and T. Fritz, Phys. Rev. B 70, 195432 (2004).

19. H. Proehl, T. Dienel, R. Nitsche, and Torsten Fritz, Phys. Rev. Lett. 93, 097403-1 (2004).

20. H. Proehl, R. Nitsche, T. Dienel, K. Leo, and T. Fritz, Phys. Rev. B 71, 165207 (2005).

21. J. F. Ziegler, J. P. Biersack, and U. Littmark, "The Stopping and Range of Ions in Solids", in Stopping and Ranges of Ions in Matter (Pergamon Press, New York, 1985), vol. I, available from www.SRIM.org (2008).

22. A. Licciardello, M. E. Fragalà, G. Foti, G. Compagnini, and O. Puglisi, Nucl. Instrum. Meth. Phys. Res. B 116, 168 (1996).

23. D. J. Li and F. Z. Cui, Bull. Mater. Sci. 21, 445 (1998). 